\title{
PHENYLKETONURIA - RESULTS OF A PILOT SURVEY
}

\author{
Major T. LIND, \\ M.B., B.S., D.Obst.R.C.O.G., R.A.M.C.
}

Specialist in Obstetrics and Gynaecology, British Military Hospital, Berlin.

IN recent years attention has been focused on the detection and subsequent control of phenylketonuria. (Gibbs and Woolf; Boyd; Hudson and Ireland). Following official instructions in 1963 on this subject, a survey was organised in Berlin which has achieved a follow-up of 99.75 per cent of all babies delivered.

Phenylketonuria is a disorder of amino-acid metabolism such that phenylalanine (derived from nitrogen-forming proteins) is prevented from forming melanine. This change is normally activated by the action of liver hydroxylase. In the absence of this latter substance the kidhey attempts to excrete the phenylalanine as phenylacetate, phenylpyruvate and phenyllactate; or more generally the phenylketones and hence the name. This disorder usually leads to mental deficiency with, in some cases, deafness and blindness. Control of dietary intake of nitrogen-forming proteins can arrest this progression and therefore its detection at the earliest possible time of of paramount importance.

Phenylketones in the urine can be detected quite simply with ferric chloride, but even more simply by 'Phenistix' (Ames and Co.). The problem arises in trying to secure the specimen of urine to test in newborn children, and deciding the time intervals at which the test should be done. Various workers have suggested different times for optimum detection of this condition. Gibbs and Woolf (1959) suggest 3 weeks and 8 weeks, Armstrong (1959) at 2 weeks and 6 weeks and Boyd (1961) was content with one test between 6 weeks and 3 months. Certainly many workers have detected a positive urine test as early as 6 days from birth. In this survey it was decided to test urine samples at 2 weeks and 4 weeks after delivery.

A survey undertaken in Cardiff (Gibbs and Woolf, 1959) had only 26 per cent success because they asked the mother to obtain a sample of baby's urine in a bottle-an almost impossible task! Boyd (1961) in Birmingham had more success (98.8 per cent) but used the district nursing services extensively and was.content with one test six weeks or more after delivery. Some workers would feel this was perhaps a little late. It was also noted that a nurse could call up to six times at one home before getting the chance to find the nappy wet, or mother at home. Hudson and Ireland (1959) pointed out that if a filter paper was soaked in urine and allowed to dry at room temperature a positive test could be obtained 6 to 10 days later. This fact was utilized in the present survey.

\section{Methods}

As each mother was instructed in feeding, bathing and general care of her child during the puerperium the midwife also explained that we wished to test baby to make sure "that he was using his food to the best advantage". Possible disease and certainly mental deficiency were never mentioned as it was felt this would cause distress. The word 'disorder' was normally used if a patient enquired directly.

Each patient was given 2 envelopes ready addressed to the pathology laboratory Each contained an $11 \mathrm{~cm}$. filter paper folded twice to become quadrant-shaped. The name of the patient was written in ink on the edge of these papers and the dates on which 
they should be used, i.e. 14 days after birth and 28 days. The papers were placed between the layers of nappy overlying the genital area. In this way they became soaked with urine and faecal staining was kept to a minimum. They were then placed on a saucer and allowed to dry. Later they would be put into the envelope provided and posted to the laboratory.

This scheme was devised with the following facts in mind :-

1. As the majority of patients have left hospital by the time the first test is required it means the person normally responsible for doing the test would have to be the doctor or nurse on district.

2. With the present shortage of all branches of the medical and nursing profession it was felt that medical officers and SSAFA sisters would find it difficult to test every child in their area twice in less than six weeks. Especially would this be so if some have to be visited six times to get one test.

3. As mother is with the child all day it seemed that some method of utilising her cooperation should be successful. As in all trials using patient co-operation however it was appreciated that 90 per cent of the work must be done before hand, i.e. addressed envelopes, etc.

4. Scientifically it is more accurate for one observer to test dozens of papers, than several observers using the 'Phenistix' in different ways.

5. Finally the scheme is run from one central area and good records can be kept with a minimum of effort. It is essential in any scheme that accurate records are kept to make sure no one is missed.

It has been suggested that the children could be tested at the SSAFA clinics, but again not all patients attend these. Also the nappy is not always conveniently wet for testing during a clinic session.

In the present survey the laboratory tested all the papers sent once per week. A 'Phenistix ' was placed on each filter paper and a drop of distilled water used to moisten both. A simple register recorded each child's date of birth and the dates the two papers arrived. This register was kept up to date on new deliveries by the maternity ward. If there was a delay in the specimens arriving the Unit R.S.M. was phoned and asked to remind the husband. If this failed, SSAFA sister was asked to call at the home. When there was time she requested the patient to send in the paper, but if not then she tested the nappy directly with 'Phenistix'.

\section{Results}

At first the scheme was slow to show results. After ten months the following figures were recorded:-

(a) Both specimens sent spontaneously

(b) First paper forgotten. Second sent spontaneously - 61 per cent

(c) First sent spontaneously, second forgotten and-

(i) Unit phoned

(ii) SSAFA sister called

(d) None sent spontaneously and-

(i) Unit phoned

(ii) SSAFA sister called
6 per cent

3 per cent - 9 per cent

15 per cent

5 per cent
7 per cent

20 per cent 
Three per cent defied all efforts, even direct calls by SSAFA sister, out of a total of 150 cases. A point of interest is that two of these children died at less than eight months of age. One was a 'cot death' and the other from measles encephalitis. Neither had evidence of phenylketonuria.

It will be seen from these figures that without any prompting at all approximately 77 per cent of all babies would be tested once before six weeks of age. This alone is a reasonable figure but a little effort achieves a higher degree of follow-up. Now the scheme is in its second year and over 400 cases have been followed. Patients now ask about the scheme, having heard of it from their friends.

For these 400 cases the figures are as follows:-

Both papers sent spontaneously

269 cases

First sent spontaneously ( 8 per cent needing to be reminded for second)

Second sent, first missed

51 cases

First late (reminded), second spontaneously

21 cases

58 cases

Thus 85.2 per cent of cases sent one specimen in spontaneously, an increase of 7 per cent. Where SSAFA sister reminded them the first was late the second paper was nearly always sent spontaneously bringing the total followed-up to 99.75 per cent tested before the sixth week of life with very little effort on anyones behalf. The outside doctors were not called upon at all and the SSAFA sisters visited only 14.5 per cent of all cases delivered.

\section{Discussion}

In this survey a relatively small number have been followed-up, but it is felt that there is sufficient evidence to show it to be a practical answer to the problem of phenylketonuria detection. When it is relying on patient's co-operation alone 75 per cent of all babies will be tested at least once before six weeks of age. With a little effort 99 per cent will be tested at least once and over 90 per cent twice. While it may be argued that in more scattered areas than Berlin the scheme will not work it is felt that in all areas served by the Army Medical Services a little bit of extra effort could achieve a follow-up comparable, if not better than, the civilian surveys done to date.

\section{Summary}

The problem of phenylketonuria detection is discussed, and a method for its detection described. The figures of a pilot survey are given to show that a high degree of followup can be achieved utilizing the co-operation of the mothers.

\section{REFERENCES}

Armstrong, et al. (1959). Chemical Pathology of the Nervous System. Pergamon Press, New York. BOYD, M. M. M. (1961). B.M.J. March 18th. p.771.

GibBs, N. K. and Woolf, L. I. (1959), B.M.J. 2, 532.

Hudson, F. P. and IRELAND, J. (1959). B.M.J. 2, 885. 